\title{
The use of oxygen reserve index in one-lung ventilation and its impact on peripheral oxygen saturation, perfusion index and, pleth variability index
}

\author{
Gonul Sagiroglu', Ayse Baysa $2^{2^{*}}$ and Yekta Altemur Karamustafaoglu ${ }^{3}$
}

\begin{abstract}
Background: Our goal is to investigate the use of the oxygen reserve index (ORi) to detect hypoxemia and its relation with parameters such as; peripheral oxygen saturation, perfusion index (PI), and pleth variability index (PVI) during one-lung ventilation (OLV).
\end{abstract}

Methods: Fifty patients undergoing general anesthesia and OLV for elective thoracic surgeries were enrolled in an observational cohort study in a tertiary care teaching hospital. All patients required OLV after a left-sided doublelumen tube insertion during intubation. The definition of hypoxemia during OLV is a peripheral oxygen saturation (SpO2) value of less than $95 \%$, while the inspired oxygen fraction ( $\mathrm{FiO} 2$ ) is higher than $50 \%$ on a pulse oximetry device. ORi, pulse oximetry, Pl, and PVI values were measured continuously. Sensitivity, specificity, positive and negative predictive values, likelihood ratios, and accuracy were calculated for ORi values equal to zero in different time points during surgery to predict hypoxemia. At Clinicaltrials.gov registry, the Registration ID is NCT05050552.

Results: Hypoxemia was observed in 19 patients (38\%). The accuracy for predicting hypoxemia during anesthesia induction at ORi value equals zero at 5 min after intubation in the supine position (DS5) showed a sensitivity of $92.3 \%$ (95\% Cl 84.9-99.6), specificity of 81.1\% (95\% Cl 70.2-91.9), and an accuracy of 84.0\% (95\% Cl 73.8-94.2). For predicting hypoxemia, ORi equals zero show good sensitivity, specificity, and statistical accuracy values for time points of DS5 until OLV30 where the sensitivity of $43.8 \%$, specificity of $64 \%$, and an accuracy of $56.1 \%$ were recorded. ORi and SpO2 correlation was found at DS5, 5 min after lateral position with two-lung ventilation (DL5) and at 10 min after OLV (OLV10) ( $p=0.044, p=0.039, p=0.011$, respectively). Time-dependent correlations also showed that; at a time point of DS5, ORi has a significant negative correlation with PI whereas, no correlations with PVI were noted.

Conclusions: During the use of OLV for thoracic surgeries, from 5 min after intubation (DS5) up to 30 min after the start of OLV, ORi provides valuable information in predicting hypoxemia defined as SpO2 less than 95\% on pulse oximeter at $\mathrm{FiO} 2$ higher than $50 \%$.

Keywords: One lung ventilation, Hypoxemia, Oxygen reserve index, Perfusion index, Pleth variability index

*Correspondence: draysebay@yahoo.com

2 Pendik District Hospital, Clinic of Anesthesiology and Reanimation, Pendik, 34980 Istanbul, Turkey

Full list of author information is available at the end of the article

\section{Introduction}

One-lung ventilation and thoracic surgeries

There is an ongoing investigation to provide advanced monitoring techniques during thoracic surgeries that require one-lung ventilation (OLV). For patients with original author(s) and the source, provide a link to the Creative Commons licence, and indicate if changes were made. The images or other third party material in this article are included in the article's Creative Commons licence, unless indicated otherwise in a credit line to the material. If material is not included in the article's Creative Commons licence and your intended use is not permitted by statutory regulation or exceeds the permitted use, you will need to obtain permission directly from the copyright holder. To view a copy of this licence, visit http://creativecommons.org/licenses/by/4.0/. The Creative Commons Public Domain Dedication waiver (http://creativeco mmons.org/publicdomain/zero/1.0/) applies to the data made available in this article, unless otherwise stated in a credit line to the data. 
a possible diagnosis of lung tumor, the surgical team performs either a video-assisted thoracoscopy (VATS) or thoracotomy surgical procedures. The anesthesiologists perform OLV in a lateral decubitus position after a double-lumen tube (DLT) insertion during tracheal intubation. There is usually a request from the surgeon for a collapsed lung where they perform the operative procedure in a surgical field. The lower, dependent lung is ventilated, whereas the upper, non-dependent lung collapses when opening the chest. There is perfusion in this lung, causing a transpulmonary shunt without ventilation. The transpulmonary shunt in the non-dependent lung is the main reason for hypoxemia during OLV. This hypoxemia in the upper deflated lung causes a physiological mechanism called hypoxic pulmonary vasoconstriction (HPV) which is responsible for diverting blood flow from the non-ventilated lung to the ventilated lung. Therefore, HPV causes a decrease in ventilation-perfusion mismatch and improves arterial oxygenation [1-4]. There are other causes of hypoxemia $[2,3,5]$. Despite the correct placement of the DLT, hypoxemia occurs in approximately 10 to $25 \%$ of patients and routine use of flexible brochoscopy for positioning of the DLT decreased the incidence of hypoxemia $[3,5]$.

\section{Definition of hypoxemia during one-lung ventilation}

The definition of hypoxemia during OLV is a peripheral oxygen saturation $\left(\mathrm{SpO}_{2}\right)$ value of less than $95 \%$ while the inspired oxygen fraction $\left(\mathrm{FiO}_{2}\right)$ is $50 \%$ or higher on a pulse oximetry device [4]. Mild hypoxemia is considered where $\mathrm{SpO}_{2}$ values are between 95 and 90\% meanwhile, arterial partial pressure of oxygen $\left(\mathrm{PaO}_{2}\right)$ values from arterial blood gas analysis show values of $75-60 \mathrm{mmHg}$. Severe hypoxemia refers to a $\mathrm{SpO}_{2}$ value of less than $90 \%$ and corresponds to $\mathrm{PaO}_{2}$ values of less than $60 \mathrm{mmHg}$ [3, 4]. A derivative of arterial oxygen saturation can be measured peripherally as $\mathrm{SpO}_{2}$ using a non-invasive monitoring device called pulse oximetry. This device measures the level of $\mathrm{PaO}_{2}$ in the range of 0 to $100 \mathrm{mmHg}$ where $\mathrm{FiO}_{2}$ value is equal to $21 \%$. However, a pulse oximetry device cannot consistently detect desaturation when $\mathrm{FiO}_{2}$ is greater than $50 \%[2,3,5]$.

\section{Pulse oximetry versus oxygen reserve index for detection of hypoxemia and hyperoxemia}

The Oxygen Reserve Index (ORi) is a multiwavelength pulse oximeter, and it provides continuous analysis of $\mathrm{PaO}_{2}$ values of moderate hyperoxia at a range of 100-200 mmHg [2-9]. This device can measure several oximeter-related parameters including; $\mathrm{ORi}, \mathrm{SpO}_{2}$, perfusion index (PI), and perfusion pleth variability (PVI). The multiwave pulse co-oximetry device can provide a calculated ORi for pulse oximetry values greater than $98 \%$. If we could give an example, it would be an incidence where a falling $\mathrm{PaO}_{2}$ value approaches $100 \mathrm{mmHg}$, and a $\mathrm{SpO}_{2}$ value is higher than $98 \%$. The multiwave oximeter device measures an ORi value that decreases and approaches a value of 0.24 [9]. This observation in a previous study provided data that ORi may provide information in both clinical situations where there is an impending hypoxic state or an unintended hyperoxic state [6-10]. ORi parameter offers a value that ranges between "1," which shows a significant oxygen reserve, to "0," which reveals no oxygen reserve. ORi begins to increase from 0.00 at a $\mathrm{PaO}_{2}$ value of $100 \mathrm{mmHg}$ and reaches a plateau of 1.00 at a $\mathrm{PaO}_{2}$ value of $200 \mathrm{mmHg}$.

\section{Other oximeter parameters: perfusion index (PI), and pleth variability index (PVI)}

$\mathrm{PI}$ is an indicator of the relative strength of the pulsatile signal from a pulse oximetry device. A higher PI value shows that the pulsatile movement increases, and peripheral circulation at the sensor site improves accordingly. The PVI is a relative variability in the pleth waveform and provides a value between 0 and 100 in a noninvasive measurement from a pulse oximetry device. PVI is an automatic measurement of the dynamic change in PI that occurs during a complete respiratory cycle $[11,12]$.

\section{Main objective of the study}

The main objective of this study is to investigate the effects of ORi parameter on hemodynamical parameters (heart rate and blood pressure) and oximeter-related parameters such as; peripheral oxygen saturation, PI, and PVI during elective thoracic surgeries requiring OLV and general anesthesia.

\section{Methods}

\section{Patients and settings}

The investigators performed a prospective observational cohort study in 14 months on patients requiring elective thoracic surgery for open lung resection via a thoracotomy or VATS at the Trakya University School of Medicine Hospital, Edirne, Turkey. The investigators conducted the study between 2020 and 2021. After the Hospital Ethics Committee (TÜTF-BAEK 2020/108), the investigators recruited patients for this clinical study. Out of a total of 59 patients, 50 patients with a diagnosis of lung tumor underwent either VATS or open thoracotomy. The surgical procedures during these operations include; either lobectomy, pneumonectomy, lung biopsy, or wedge resection. The Human Research Ethics Committee of Trakya University Medical Faculty, Edirne, Turkey approved this clinical study protocol. The investigators collected written informed consent from patients or their relatives for this clinical study during preoperative visits. 
The study is registered in the Clinicaltrials.gov registry, and our Registration ID is NCT05050552. The pulmonary function tests, including the percentage of expected, forced expired volume during the first second $\left(\mathrm{FEV}_{1} \%\right)$, the ratio of $\mathrm{FEV}_{1} / \mathrm{FVC} \%$ (percentage of expected forced vital capacity to $\mathrm{FEV}_{1}$ ) were done in some patients with a possible diagnosis of severe lung disease because of the global pandemia in 2020 and 2021. Patients with $\mathrm{FEV}_{1}$ between 30 and $80 \%$ and $\mathrm{FEV}_{1} / \mathrm{FVC}$ ratio of $<70 \%$ were considered as having a moderate level of chronic obstructive pulmonary disease as per literature. These patients were included whereas, severely restricted patients were excluded $[2,3]$.

Inclusion criteria included; patients at ages between 22 and 80 years old, American Society of Anesthesiologists Physical Status (ASA-PS) risk groups of 1 to 3, surgical procedures of either open lung resection with thoracotomy or VATS, general anesthesia including sevoflurane inhalational anesthesia during maintanence, the use of DLT and OLV. Exclusion criteria include; refusal to participate in a study, history of severe asthma, preoperative renal insufficiency (creatinine $>114 \mathrm{umol} / \mathrm{L}$ ); preoperative liver dysfunction (aspartate amino transferaseAST $>40 \mathrm{U} / \mathrm{L}$, alanine amino transferase-ALT $>40 \mathrm{U} / \mathrm{L}$ ); previous history of coronary or vascular disease or heart failure with an ejection fraction less than $40 \%$, lung function study showing an $\mathrm{FEV}_{1}$ less than $50 \%$, history of severe chronic respiratory disease of the non-operated lung, pregnancy, history of previous pulmonary resection and hemoglobinopathies $[8,9,13]$.

\section{The anesthetic management, definition of hypoxemia and collected data during OLV}

The investigators did not administer drugs for premedication to prevent hypoxemia-related events. After admitting a patient to the operating theatre, anesthesiologists applied electrocardiogram, noninvasive blood pressure and pulse oximetry monitoring devices, and measured these parameters continuously. The monitored parameters include; heart rate (HR), mean arterial pressure (MAP), systolic blood pressure (SBP), diastolic blood pressure (DBP), and $\mathrm{SpO}_{2}$. The anesthesiologists provided general anesthesia using intravenous doses of propofol (Pofol, Fresenius Pharmaceutical, Turkey), 2 to $3 \mathrm{mg} / \mathrm{kg}$, rocuronium (Esmeron, Organon Pharmaceuticals, USA) at a dose of $0.6 \mathrm{mg} / \mathrm{kg}$, and fentanyl (Janssen fentanyl, Janssen Pharmaceutical, Belgium) at a dose of 2 to $3 \mathrm{mcg} / \mathrm{kg}$. The anesthesiologist placed a 20 Gauge radial artery catheter on all patients and connected it to a disposable pressure transducer to provide continuous monitoring following the induction of anesthesia. During tracheal intubation, a left Robertshaw DLT was used. The anesthesiologist used a flexible broncoscopy for correct positioning of DLT in supine and lateral decubitus positioning. For anesthetic maintenance, anesthesiologists used inhalational anesthetic of sevoflurane (Sevorane, Abbott Pharmaceutical, USA) at an end-tidal concentration of 1 to $2 \%$ and intravenous fentanyl boluses at a dose of 0.5 to $1 \mathrm{microgram} / \mathrm{kg}$ every hour. The hemodynamical stability was maintained during the surgical procedures where keeping HR between 60 and 100 beats/minute and keeping MAP between 60 and $80 \mathrm{mmHg}$. During surgery, intravenous rocuronium was used every hourly at a dose of $0.05 \mathrm{mg} / \mathrm{kg}$. All patients received an intravenous infusion of lactated Ringer's solution at a dose of $10 \mathrm{ml} / \mathrm{kg} / \mathrm{hr}$.

Hemodynamical and oximeter-related data of $\mathrm{HR}$, MAP, SBP, DBP, $\mathrm{SpO}_{2}, \mathrm{PaO}_{2}$, ORi, PI, and PVI values were recorded at thirteen different time points during anesthesia induction and maintenance of the surgery. Radical-7 Pulse CO-Oximeter is used to measure oximeter parameters of ORi, PI, and PVI (Masimo Inc., Irvine, CA, USA). During the collection of these parameters, the investigators measured peripheral oxygen saturation using a Pulse CO-Oximetry probe. For other oximeterrelated parameters, the Rainbow R1 25-L probe was used, a product of the same company [8,9]. Baseline values of ORi provide data before preoxygenation, and afterward, patients were pre-oxygenated with $100 \%$ oxygen. Therefore, the list of time points for collection of data include as follows; first, during the patient's arrival to the operating room in the supine position breathing room air (basal), during preoxygenation with $100 \%$ oxygen in the supine position (preoxygenation), $5 \mathrm{~min}$ after tracheal intubation during two-lung ventilation in the supine position (ORiDS5), 5 min after placing the patient in a lateral position with two-lung ventilation (ORiDL5), at $1 \mathrm{~min}$ after OLV placement (OROLV1), and afterwards; at $2 \mathrm{~min}$ (OROLV120), $5 \mathrm{~min}$ (OROLV5), $10 \mathrm{~min}$ (OROLV10), $15 \mathrm{~min}$ (OROLV15), $30 \mathrm{~min}$ (OROLV30), $45 \mathrm{~min}$ (OROLV45), $60 \mathrm{~min}$ (OROLV60) and $90 \mathrm{~min}$ after OLV placement (OROLV90) [8, 9, 13, 14].

After general anesthesia induction and intubation, the anesthesiologists provided mechanical ventilation, and two lung ventilation in the supine position required the settings of a tidal volume of $8-10 \mathrm{~mL} / \mathrm{kg}$, inspiration to expiration ratio of $1: 2$, and respiratory rate of $10-12 / \mathrm{min}$, without positive end-expiratory pressure (PEEP). During operation, the surgical team provided a lateral decubitus position before incision and the anesthesiologist initiated OLV after positioning. The dependent lung was ventilated with a tidal volume of $6-8 \mathrm{~mL} / \mathrm{kg}$, I: E ratio of $1: 2$, respiratory rate of $12-14 / \mathrm{min}$ with an unchanged $\mathrm{FiO}_{2}$ of 0.5 with an Aestiva 3000 ventilator (Datex-Ohmeda Inc. Madison, U.S.A.) [6, 15]. During surgery, the anesthesiologists were responsible for the anesthesia maintenance with the use of anesthetic agents such as; inhalational 
anesthesia of sevoflurane, intravenous rocuronium maintenance dose of $0.05 \mathrm{mg} / \mathrm{kg}$ every hourly, and intravenous fentanyl maintenance dose of 1 to $2 \mathrm{mcg} / \mathrm{kg}$.

Hypoxemia during OLV is a $\mathrm{SpO}_{2}$ value of less than $95 \%$ while the $\mathrm{FiO}_{2}$ is $50 \%$ or greater on a pulse oximetry device $[4,5,9]$. The anesthesiologist who conducts the anesthesia during surgery was responsible for increasing $\mathrm{FiO}_{2}$, using bag-mask ventilation of $100 \%$ for a while, implementing an alveolar recruitment maneuver, or using continuous positive airway pressure to the collapsed lung during a desaturation of $\mathrm{SpO}_{2}$ value less than $95 \%[2,3,8$, $9,11,13]$. A flexible broncoscopy was present during the whole surgical procedure to detect malpositioning of the DLT. The investigators recorded the duration of surgery, anesthesia, and duration of OLV.

\section{The management of hypoxemic events and other unwanted events during surgery}

The anesthesiologists provided oxygen titration depending mainly on the $\mathrm{SpO}_{2}$ values in our study group of patients. The data collectors were usual residents in anesthesiology. The residents performed a blood gas analysis at DL5 time point only. The reason for the abscence of this routine arterial blood gas analysis during thoracic surgeries was a recent colloborative decision of our hospital and anesthesiology department to decrease medical costs. In addition, although arterial blood gases analysis is crucial to document the exact measurement of oxygenation via $\mathrm{PaO}_{2}$ values, it is impractical to obtain real-time values during an episode of hypoxemia $[8,9]$.

After induction, patients were routinely ventilated with $50 \% \mathrm{FiO}_{2}(50 \%$ oxygen $+50 \%$ air mixture, 11 /minute fresh gas flow). The anesthesiologist was responsible for keeping $\mathrm{SpO}_{2}$ values greater than 94. For this purpose, necessary adjustments in $\mathrm{FiO}_{2}$ values and mechanical ventilation parameters as well as necessary maneuvers were performed to provide better oxygenation. The incidence of thromboembolic complications, arrhythmias, pneumonia, the duration of hospital and intensive care unit stay were recorded [9, 11, 13-18]. Intravenous ephedrine (Ephedrine, Osel Pharmaceutical, Turkey) at a dose of $10 \mathrm{mg}$ bolus injections were considered if SBP was less than $90 \mathrm{mmHg}$. Hypotension was defined as a decrease in MAP more significant than $20 \%$ after anesthesia induction and treated with intermittent bolus doses of $5 \mathrm{mg}$ ephedrine. The definition of hypotension was based on previous studies [12].

\section{Summary of surgical procedure}

Surgical resection was performed through a posterolateral thoracotomy. A suspicious tumor was located, and if possible all necessary frozen section samples were obtained for pathological evaluation. At the end of the operation, the suspicious mass was removed from its location. The necessary suturing, aspiration, and irrigation of fluids and blood were performed $[14,15,18]$.

\section{The ethical considerations}

Trakya University Faculty of Medicine University Ethical Committee agreed and approved the study in February 2020. All patients approved the fully informed written consent to participate in the study. The participants had confidentially during the study process and were able to withdraw from the research process at any time. The investigators discussed any expected benefits or potential harm for the research in detail.

\section{Statistical analysis}

The investigators used an SPSS 15.0 (Statistical Package for Sciences, USA) program to analyze the data of our clinical study. Data were presented as mean \pm SD and numbers (percentages), as indicated. Normality was tested with the Kolmogorov-Smirnov test. Some parameters are reported as median (interquartile range [IQR], 25 th to 75 th percentile). Sensibility, specificity, positive and negative predicted values, likelihood ratios, and their respective confidence intervals were obtained from a two-by-two contingency table for the validity of ORi equals to zero during different moments before and after OLV was achieved to predict the first hypoxemia $\left(\mathrm{SpO}_{2}\right.$ value of $<95 \%)$ episode after $\operatorname{OLV}[8,9,13]$. The proportion of true positives and true negatives in all evaluated cases was considered to be accurate. The level of statistical significance was a $p$-value of less than 0.05 . For calculation of sample size, a hypoxemia rate of $30 \%$ after OLV, and a $10 \%$ precision at $95 \%$ confidence intervals, an alpha error of 0.05 , and a power of $80 \%$, the number of patients for the study was calculated as 28 patients $[8,13,14]$.

\section{Results}

The investigators performed the clinical study on 50 patients in 14 months duration. The median age of the whole group was 53 years (22-80). There were 28 males and 22 females. The data presented in Table 1 provides demographic information, co-morbidities, pulmonary function tests of 26 patients with possible moderate to severe lung disorders, surgical approach and type of surgery. Pulmonary function tests were not obtained from all patients due to the COVID-19 pandemic. Hemodynamic and oximeter data that are described in methods section were continuously monitored and collected at several phases of anesthesia and surgery. The residents performed arterial blood gas analysis at only one time point which is DL5 time. The residents were responsible to record pulse oximetry and other oximeter values for detection of hypoxemic episodes. 
Table 1 Demographic data and operation characteristics of undergoing elective thoracic surgery with open lung ventilation

\begin{tabular}{|c|c|}
\hline Age, (year) & $55.46 \pm 13.85$ \\
\hline Height, (cm) & $168.5 \pm 8.43$ \\
\hline Weight, (kg) & $77.76 \pm 16.1$ \\
\hline Body mass index, (kg/m2) & $27.54 \pm 6.17$ \\
\hline \multicolumn{2}{|l|}{ Gender, n (\%) } \\
\hline Female & $22(44)$ \\
\hline Male & $28(56)$ \\
\hline \multicolumn{2}{|l|}{ ASA-PS, n (\%) } \\
\hline 1 & $5(10)$ \\
\hline$\|$ & $27,854)$ \\
\hline III & $18(36)$ \\
\hline $\mathrm{FVC},(\mathrm{mL})$ & $2.87 \pm 0.68$ \\
\hline FEV1 & $2.3 \pm 0.59$ \\
\hline Smoking, n (\%) & $34(68)$ \\
\hline COPD, n (\%) & $11(22)$ \\
\hline Hypertension, n (\%) & $17(34)$ \\
\hline Diabetes mellitus, n (\%) & $8(16)$ \\
\hline Coronary artery disease, n (\%) & $6(12)$ \\
\hline Right side intervention, n (\%) & $24(48)$ \\
\hline \multicolumn{2}{|l|}{ Surgical approach, n (\%) } \\
\hline Thoracotomy & $27(54)$ \\
\hline VATS & $23(46)$ \\
\hline \multicolumn{2}{|l|}{ Type of surgery, n (\%) } \\
\hline Lung biopsy & $12(24)$ \\
\hline Wedge resection & $19(38)$ \\
\hline Lobectomy & $14(28)$ \\
\hline Pneumonectomy & $5(10)$ \\
\hline Duration of operation, (min) & $71.3 \pm 37.59$ \\
\hline
\end{tabular}

ASA-PS American Society of Anesthesiologists-physical status, BMI Body mass index, COPD Chronic obstructive pulmonary disease, FVC Forced vital capacity, FEV Forced expiratory volume fist second, VATS Video assisted; thoracoscopic surgery

Table 2 shows the data analysis of ORi equals to 0 for predicting hypoxemia at different time points during anesthesia induction and maintenance. The accuracy for predicting hypoxemia during anesthesia induction at ORi value equals zero at DS5 showed a sensitivity of $92.3 \%$ (95\% CI 84.9-99.6), specificity of $81.1 \%$ (95\% CI $70.2-$ 91.9), and an accuracy of $84.0 \%$ (95\% CI 73.8-94.2).

The accuracy for predicting hypoxemia during anesthesia induction at ORi equals zero at $5 \mathrm{~min}$ after placing the patient in a ORiDL5 showed a sensitivity of $69.2 \%$, specificity of $83.3 \%$, and an accuracy of $76.0 \%$. The 95\% confidence interval (CI) values are presented in Table 2. In this table, the data analysis shows that; for predicting hypoxemia, ORi equals to zero show good sensitivity, specificity and accuracy statistical values for time points of DS5 until OLV30 where sensitivity of $43.8 \%$, specificity of $64 \%$, and an accuracy of $56.1 \%$ were recorded. These findings correlated to the previous reports that HPV increases and intrapulmonary shunting decreases after the start of OLV within 30 to $60 \mathrm{~min}[4,8,13,14]$.

Overall, from a total of 50 patients in the study group, 19 patients (38\%) developed hypoxemia defined as $\mathrm{SpO}_{2}$ values of less than $95 \%$ at or higher than $\mathrm{FiO}_{2}$ value of $50 \%$ during the surgical procedure. At the time point of DS5, ORi equals to 0 value was observed in 12 of the 19 patients $(63.16 \%)$ who presented with hypoxemia. At other time points this hypoxemia was observed as follows; DL5; 11 patients (22\%), OLV1; 8 patients (16\%), OLV2; 9 patients (18\%), OLV5 12 patients (24\%) and OLV10 15 patients (30\%).

In Fig. 1, data analysis provides representative trends of ORi and $\mathrm{SpO}_{2}$ values in a continuous graph at thirteen different time points during the anesthesia induction and maintenance of the surgery. This correlation showed that; a strong correlation between ORi and $\mathrm{SpO}_{2}$ was found at time points of DS5 $(r=0.286, p=0.044)$, DL5 $(r=0.293, p=0.039)$, and, at OLV10 $(r=0.360$, $p=0.011)$. Therefore, Fig. 1 also supports the relationship between $\mathrm{SpO}_{2}$ values and ORi equals to zero values for predicting hypoxemia during anesthesia induction and maintanence.

Later, we evaluated the representative trends of the ORi and PI values and the ORi and PVI values at different time points during anesthesia induction and maintenance of thoracic surgeries. These are represented in Figs. 2 and 3.

For hemodynamical and oximeter parameters including; $\mathrm{HR}, \mathrm{MAP}, \mathrm{SBP}, \mathrm{DBP}, \mathrm{SpO}_{2}$ values, a correlation between these parameters were not found in the statistical analysis $(p>0.05)$. In our study, we demonstrated a time-dependent correlation between PVI and MAP at the time point of OLV90, indicating that PVI showed a relation to MAP at a late stage of the thoracic surgical procedure.

In our study, we investigated the ORi and PVI values at different time points during anesthesia induction and maintenance of thoracic surgery and our findings show that fluid deficit or fluid overload causes changes in PI and PVI values. This is observed in our representative trend graphs in Figs. 2 and 3. Our study provides valuable data for the investigation of correlations between ORi and PI, and PVI. Our study provides data that at a time point of DS5, there is a significant negative correlation with PI $(r=-0.332, p=0.019)$, whereas; no correlations with PVI were noted.

Table 3 shows the median values and interquartile range of PI and PVI values at different measurement points during the study. The analysis of correlations between these PI and PVI values showed a correlation between PI and PVI values at the time point of ORiDL5 
Table 2 The data analysis of ORi equals to zero and accuracy for predicting hypoxemia during OLV at different time points of surgery

\begin{tabular}{|c|c|c|c|c|c|c|c|}
\hline & Sensitivity & Specificity & PPV & NPV & PLHR & NLHR & Accuracy \\
\hline Preoxygenation (95\% Cl) & $0.15(0.1-0.3)$ & $91.9(84.3-99.5)$ & $40(26.4-53.6)$ & $75.6(63.6-87.5)$ & $1.9(1.9-5.7)$ & $0.9(0.8-1)$ & $72(59.6-84.4)$ \\
\hline $\begin{array}{l}\text { ORIDS5 }=0 \\
(95 \% \mathrm{CI})\end{array}$ & $92.3(84.9-99.6)$ & $81.1(70.2-.91 .9)$ & $63.2(49.8-76.5)$ & $96.8(91.9-100)$ & $4.9(1.1-10.9)$ & $0.1(0.1-0.2)$ & $84(73.8-94.2)$ \\
\hline $\begin{array}{l}\text { ORIDL5=0 } \\
(95 \% \mathrm{CI})\end{array}$ & $69.2(56.4-82)$ & $83.3(73-93.7)$ & $81.8(71.1-92.5)$ & $71.4(58.9-84)$ & $4.2(1.4-9.7)$ & $0.4(0.2-0.5)$ & $76(64.2-87.8)$ \\
\hline $\begin{array}{l}\text { OROLV1 }=0 \\
(95 \% \mathrm{Cl})\end{array}$ & $63.6(50.3-77)$ & $75(63-87)$ & $66.7(53.6-79.7)$ & $72.4(60-84.8)$ & $2.6(1.8-6.9)$ & $0.5(0.3-0.6)$ & $70(57.3-82.7)$ \\
\hline $\begin{array}{l}\text { OROLV2 }=0 \\
(95 \% \mathrm{CI})\end{array}$ & $65.2(52-78.4)$ & $70.4(57.7-83)$ & $68.2(55.3-81.1)$ & $70.4(57.7-83)$ & $2.2(1.9-6.2)$ & $0.5(0.4-0.6)$ & $69.4(56.6-82.2)$ \\
\hline $\begin{array}{l}\text { OROLV5 }=0 \\
(95 \% \mathrm{Cl})\end{array}$ & $56.5(42.8-70.3)$ & $66.7(53.6-79.7)$ & $59.1(45.5-72.7)$ & $64.3(51-77.6)$ & $1.7(0.7-2.7)$ & $0.7(0.5-0.8)$ & $62(48.5-75.5)$ \\
\hline $\begin{array}{l}\text { OROLV10=0 } \\
(95 \% \mathrm{Cl})\end{array}$ & $56(42.2-70)$ & $64(50.7-77.3)$ & $60.9(47.3-74.4)$ & $59.3(50-72.9)$ & $1.6(0.6-2.6)$ & $0.7(0.6-0.8)$ & $60(46.4-73.6)$ \\
\hline $\begin{array}{l}\text { OROLV15=0 } \\
(95 \% \mathrm{Cl})\end{array}$ & $52.2(38-66.3)$ & $68(54.8-81.2)$ & $60(46.1-73.9)$ & $60.7(46.9-74.5)$ & $1.6(0.6-2.7)$ & $0.7(0.6-0.8)$ & $60.4(46.6-74.3)$ \\
\hline $\begin{array}{l}\text { OROLV30=0 } \\
(95 \% \text { Cl) }\end{array}$ & $43.8(29.7-57.8)$ & $64(50.4-77.6)$ & $43.8(29.7-57.8)$ & $64(50.4-77.6)$ & $1.2(0.2-2.2)$ & $0.9(0.8-1)$ & $56.1(42.1-70.1)$ \\
\hline $\begin{array}{l}\text { OROLV45 =0 } \\
(95 \% \mathrm{Cl})\end{array}$ & $40(23.3-56.7)$ & $72.2(57-87.5)$ & $54.5(37.6-71.5)$ & $59.1(42.3-75.9)$ & $1.4(0.3-2.7)$ & $0.8(0.7-1)$ & $57.6(40.7-74.4)$ \\
\hline $\begin{array}{l}\text { OROLV60 }=0 \\
(95 \% \mathrm{Cl})\end{array}$ & $53.3(35.8-70.9)$ & $68.8(52.4-85.1)$ & $61.5(44.4-78.7)$ & $61.1(43.9-78.3)$ & $1.7(0.4-3)$ & $0.7(0.5-0.8)$ & $61.3(44.1-78.4)$ \\
\hline $\begin{array}{l}\text { OROLV90 }=0 \\
(95 \% \mathrm{Cl})\end{array}$ & $50(25.5-75)$ & 66.7 (43.6-90) & $71.4(49.3-93.6)$ & $44.4(20.1-68.8)$ & $1.5(0.2-4.3)$ & $0.8(0.5-0.9)$ & $56.3(31.9-80.6)$ \\
\hline
\end{tabular}

ORi Oxygen reserve index, OR Oxygen reserve, OLV One-lung ventilation, PPV Positive predictive value, NPV Negative predictive value, PLHR Positive likelihood ratio, NLHR Negative likelihood ratio, CI Confidental interval, ORiDS5 ORi under mechanical ventilation 5 min after intubation in supine position, ORiDL5 ORi under mechanical ventilation $5 \mathrm{~min}$ after positioning in the lateral decubitus position, OROLV1 ORi after 1 min of OLV, OROLV2 ORi after 2 min of OLV, OROLV 5 ORi after 5 min of OLV, OROLV10 ORi after $10 \mathrm{~min}$ of OLV, OROLV15 ORi after $15 \mathrm{~min}$ of OLV, OROLV30 ORi after $30 \mathrm{~min}$ of OLV, OROLV45 ORi after $45 \mathrm{~min}$ of OLV, OROLV60 ORi after $60 \mathrm{~min}$ of OLV, OROLV90 ORi after $90 \mathrm{~min}$ of OLV

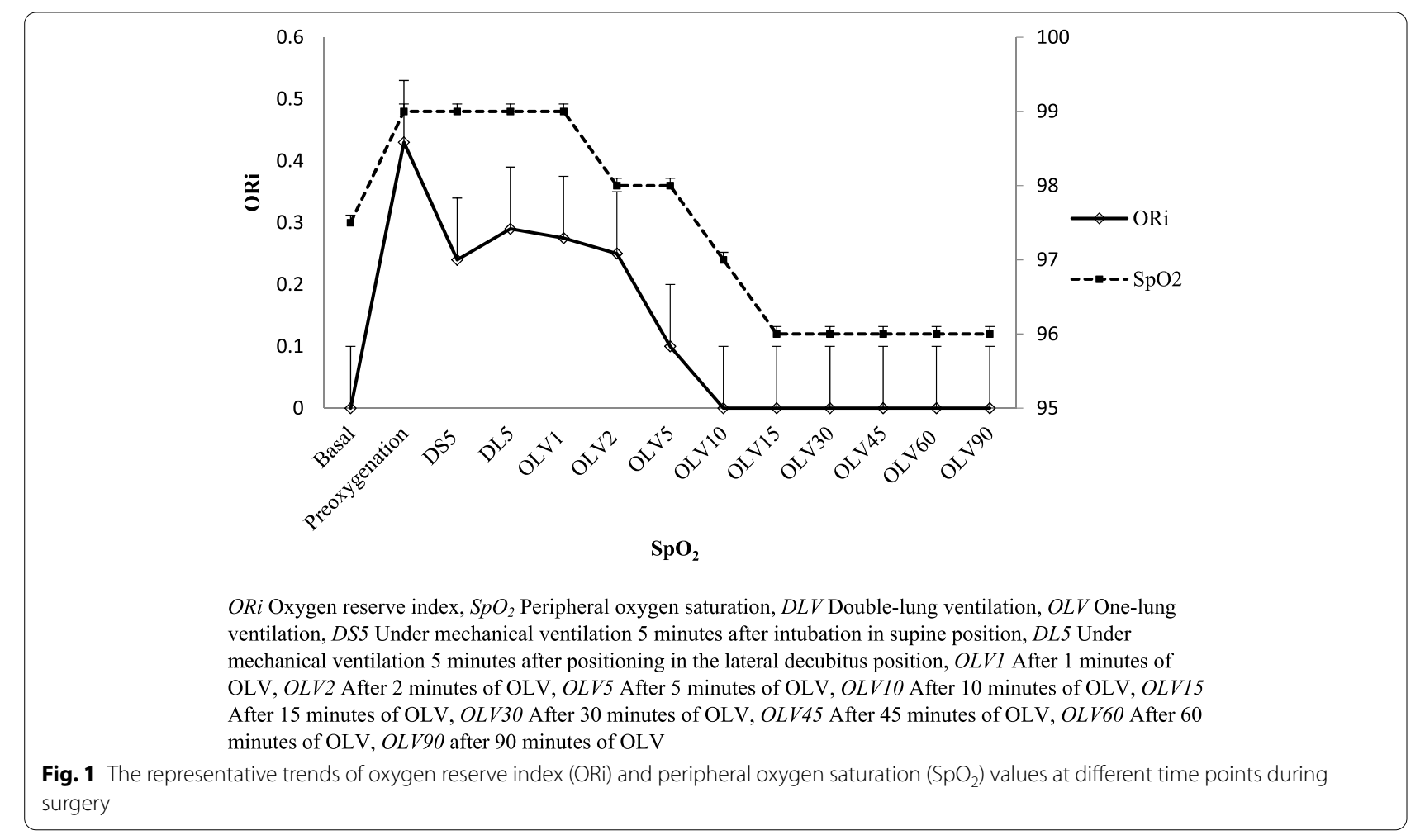




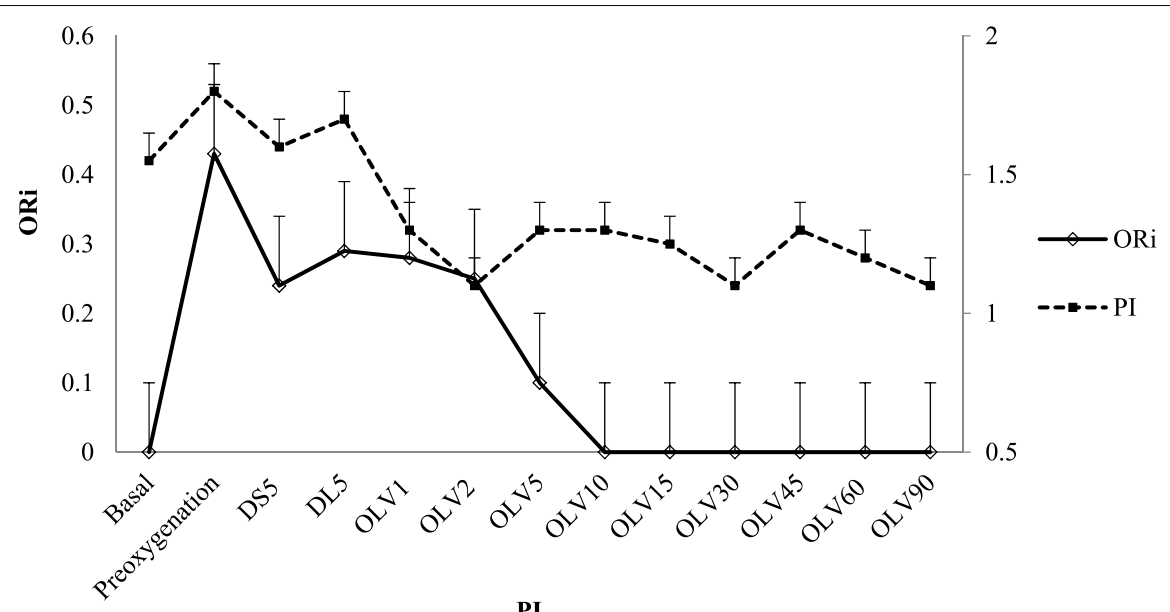

PI

ORI Oxygen reserve index, PI Perfusion index; $D L V$ Double-lung ventilation, $O L V$ One-lung ventilation, $D S 5$ Under mechanical ventilation 5 minutes after intubation in supine position, $D L 5$ Under mechanical ventilation 5 minutes after positioning in the lateral decubitus position, OLV1 After 1 minutes of OLV, OLV2 After 2 minutes of OLV, OLV5 After 5 minutes of OLV, OLV10 After 10 minutes of OLV, OLV15 After 15 minutes of OLV, OLV30 After 30 minutes of OLV, OLV45 After 45 minutes of OLV, OLV60 After 60 minutes of OLV, OLV 90 after 90 minutes of OLV

Fig. 2 The oxygen reserve index (ORi) and perfusion index (PI) values at different time points of surgery

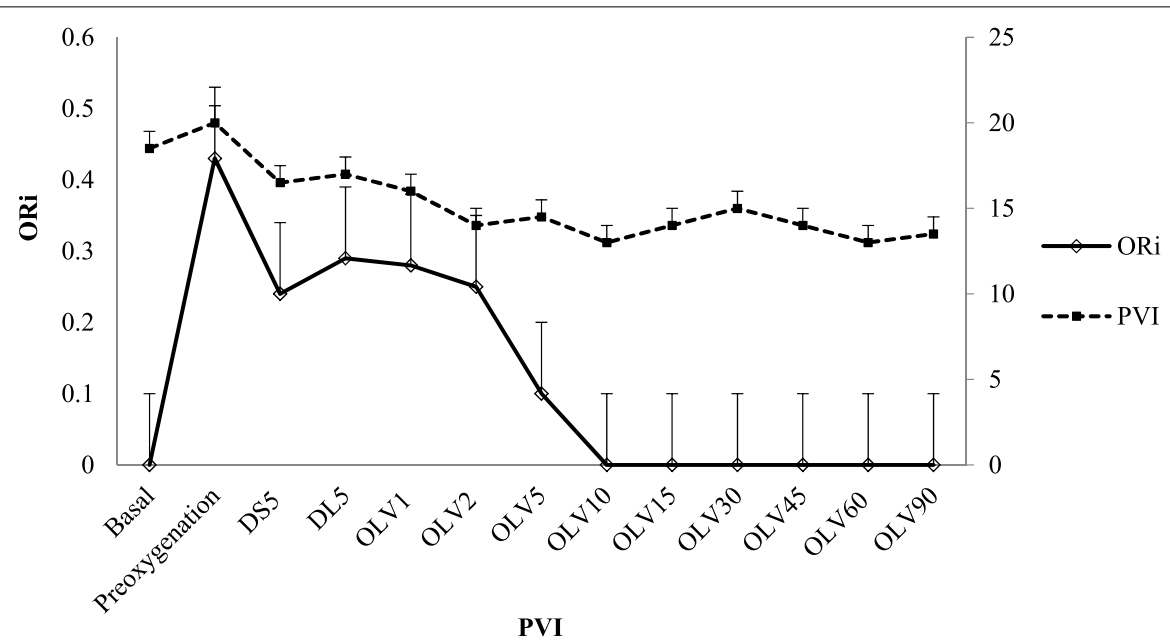

ORi Oxygen reserve index, $P V I$ Pleth variability index; $D L V$ Double-lung ventilation, $O L V$ One-lung ventilation, $D S 5$ Under mechanical ventilation 5 minutes after intubation in supine position, $D L 5$ Under mechanical ventilation 5 minutes after positioning in the lateral decubitus position, $O L V 1$ After 1 minutes of OLV, OLV2 After 2 minutes of OLV, OLV5 After 5 minutes of OLV, OLV10 After 10 minutes of OLV, OLV15 After 15 minutes of OLV, OLV30 After 30 minutes of OLV, OLV45 After 45 minutes of OLV, OLV60 After 60 minutes of OLV, OLV 90 after 90 minutes of OLV

Fig. 3 The oxygen reserve index (ORi) and pleth variability index (PVI) values at different time points of surgery

$(r=-0.284, p=0.046)$. In other time points, correlations were not demonstrated $(p>0.05)$.

Table 4 provides time-dependent correlations between ORi with $\mathrm{SpO}_{2}$, PI, and PVI. These correlation analysis provide data that ORi has significant correlations with
$\mathrm{SpO}_{2}$, PI and PVI at some specific time points and these include; at time point of DS5; $(r=0.286, p=0.044)$, DL5 $(r=0.293, p=0.039)$, and OLV10; ORi has a significant correlation with $\mathrm{SpO}_{2}(r=0.360, p=0.011)$, at time point of DLS5; ORi has a significant negative correlation with 
Table 3 The median values and interquartile range of perfusion index (PI) and pleth variability index (PVI) values at different measurement points of surgery

\begin{tabular}{|c|c|c|c|c|}
\hline \multirow[t]{2}{*}{ Time (min) } & \multicolumn{2}{|c|}{ Perfusion Index (PI) } & \multicolumn{2}{|c|}{$\begin{array}{l}\text { Pleth Variability Index } \\
\text { (PVI) }\end{array}$} \\
\hline & Median & $\begin{array}{l}\text { Interquartile } \\
\text { range (IQR) }\end{array}$ & Median & $\begin{array}{l}\text { Interquartile } \\
\text { range (IQR) }\end{array}$ \\
\hline Baseline & 1.55 & $0.86-2.3$ & 20.5 & $14-30.25$ \\
\hline Preoxygenation & 1.8 & $1.3-2.6$ & 18.5 & $13-30.25$ \\
\hline DS5 & 1.6 & $1-2.5$ & 16 & $11-21$ \\
\hline DL5 & 1.7 & $1.28-2.3$ & 17 & $12-26$ \\
\hline OLV1 & 1.3 & $0.61-1.3$ & 16.5 & $11.75-23$ \\
\hline OLV2 & 1.1 & $0.63-1.93$ & 13.5 & $10-21.25$ \\
\hline OLV5 & 1.3 & $0.64-1.93$ & 14 & $10-20.25$ \\
\hline OLV10 & 1.3 & $0.71-1.7$ & 17 & $10.5-22.5$ \\
\hline OLV15 & 1.25 & $0.76-2.1$ & 15 & $10.25-21$ \\
\hline OLV30 & 1.1 & $0.66-2$ & 17 & $10-22$ \\
\hline OLV45 & 1.3 & $0.82-2.1$ & 14 & $8.5-20.5$ \\
\hline OLV60 & 1.2 & $0.63-2.2$ & 14 & $10-22$ \\
\hline OLV90 & 1.1 & $0.73-2$ & 13 & $8.5-18.75$ \\
\hline
\end{tabular}

PI Perfusion index, PVI Pleth variability index, IQR Interquartile range, DLV Double-lung ventilation, OLV One-lung ventilation, DS5 Under mechanical ventilation $5 \mathrm{~min}$ after intubation in supine position, DL5 Under mechanical ventilation $5 \mathrm{~min}$ after positioning in the lateral decubitus position, OLV1 After 1 min of OLV, OLV2 After 2 min of OLV, OLV5 After 5 min of OLV, OLV10 After $10 \mathrm{~min}$ of OLV, OLV15 After $15 \mathrm{~min}$ of OLV, OLV30 After $30 \mathrm{~min}$ of OLV, OLV45 After $45 \mathrm{~min}$ of OLV, OLV60 After $60 \mathrm{~min}$ of OLV, OLV90 after $90 \mathrm{~min}$ of OLV
PI $(r=-0.332, p=0.019)$, whereas; 3 - no correlations with PVI was noted.

\section{Discussion}

The main findings of this study are provided below:

The main conclusion is that ORi is sensitive and specific in predicting hypoxemia defined as $\mathrm{SpO}_{2}$ values of less than $95 \%$ while the $\mathrm{FiO}_{2}$ is $50 \%$ or higher on a pulse oximetry device at $5 \mathrm{~min}$ after intubation in the supine position (sensitivity of $92.3 \%$, specificity of $81.1 \%$ and, an accuracy of $84.0 \%$ ) [7-9, 13, 15, 17-21].

There are other time points where there is statistically good report of sensitivity, specificity and accuracy for time points at ORiDL5, and during OLV until OLV30 where sensitivity of $43.8 \%$, specificity of $64 \%$, and an accuracy of $56.1 \%$ are recorded. These findings correlated to the previous reports that HPV increases and intrapulmonary shunting decreases after the start of OLV within 30 to $60 \mathrm{~min}[4,8,13,14]$.

In our study group of patients, a total of 19 patients (38\%) developed hypoxemia at various recorded time points during the surgical procedure. ORi provides information for impending hypoxemia that a change in ORi value can be detected 5 to $6 \mathrm{~min}$ earlier than pulse oximetry value. Therefore, ORi can provide a valuable time to the anesthesiologist to provide an increase in $\mathrm{FiO}_{2}$ values, to perform necessary mechanical ventilation adjustments, to perform aspiration or other anesthetic management techniques to prevent hypoxemia $[7-9,13$, $15,17-21]$.

Table 4 Time-dependent correlations between oxygen reserve index (ORi) with peripheral oxygen saturation $\left(\mathrm{SpO}_{2}\right)$, perfusion index (PI) and pleth variability index (PVI) during surgery

\begin{tabular}{|c|c|c|c|c|c|c|}
\hline \multirow[t]{2}{*}{ Time (min) } & \multicolumn{2}{|c|}{ Peripheral Oxygen Saturation $\left(\mathrm{SpO}_{2}\right)$} & \multicolumn{2}{|c|}{ Perfusion Index (PI) } & \multicolumn{2}{|c|}{$\begin{array}{l}\text { Pleth Variability Index } \\
\text { (PVI) }\end{array}$} \\
\hline & $r$ & $p$ & $r$ & $P$ & $r$ & $p$ \\
\hline Preoxygenation & 0.121 & 0.404 & 0.042 & 0.774 & 0.017 & 0.908 \\
\hline DS5 & 0.286 & $0.044^{*}$ & -0.332 & $0.019^{*}$ & 0.073 & 0.617 \\
\hline DL5 & 0.293 & $0.039^{*}$ & -0.010 & 0.947 & 0.089 & 0.540 \\
\hline OLV1 & -0.030 & 0.834 & 0.020 & 0.888 & -0.013 & 0.984 \\
\hline OLV2 & -0.087 & 0.548 & 0.158 & 0.272 & -0.147 & 0.307 \\
\hline OLV5 & -0.249 & 0.081 & 0.133 & 0.358 & -0.001 & 0.997 \\
\hline OLV10 & 0.360 & $0.011^{*}$ & -0.240 & 0.097 & -0.058 & 0.692 \\
\hline OLV15 & 0.241 & 0.099 & $-0,247$ & 0.091 & -0.175 & 0.234 \\
\hline OLV30 & -0.162 & 0.313 & 0.305 & 0.053 & -0.189 & 0.237 \\
\hline OLV45 & 0.270 & 0.129 & -0.115 & 0.529 & 0.038 & 0.837 \\
\hline OLV60 & 0.092 & 0.630 & -0.179 & 0.344 & -0.036 & 0.850 \\
\hline OLV90 & $-0,412$ & 0.113 & 0.433 & 0.094 & -0.167 & 0.535 \\
\hline
\end{tabular}

${ }^{*}$ A p-value of less than 0.05 is considered statistically significant.

ORi Oxygen reserve index, $\mathrm{SpO}_{2}$ Peripheral oxygen saturation, $\mathrm{PI}$ Perfusion index, PVI Pleth variability index, DLV Double-lung ventilation, OLV One-lung ventilation, DS5 Under mechanical ventilation $5 \mathrm{~min}$ after intubation in supine position, DL5 Under mechanical ventilation 5 min after positioning in the lateral decubitus position, OLV1 After 1 min of OLV, OLV2 After 2 min of OLV, OLV5 After 5 min of OLV, OLV10 After 10 min of OLV, OLV15 After 15 min of OLV, OLV30 After 30 min of OLV, OLV45 After $45 \mathrm{~min}$ of OLV, OLV60 After $60 \mathrm{~min}$ of OLV, OLV90 after $90 \mathrm{~min}$ of OLV 
During OLV, hypoxemia can develop not only by the intrapulmonary shunt in the non-ventilated lung but also by the ventilation-perfusion mismatch in the ventilated lung or hemodynamic instability $[4,5]$. In our study, patients with coronary artery disease and an ejection fraction below $40 \%$ were not included into the study. Patients with heart failure were also excluded. During OLV, atelectasis occurs during general anesthesia induction, which causes ventilation/perfusion mismatch even before switching to OLV $[5,6,10]$. During OLV, oxygen delivery to the patient under general anesthesia occurs during various interactions between hemoglobin, oxygen saturation, cardiac output, and normal physiological mechanisms such as HPV and intrapulmonary shunts $[3,4]$. Although the causes of OLV-induced hypoxemia are multifactorial, early detection of hypoxemia before the onset of OLV allows the application of different ventilation strategies to improve oxygenation [3-6]. The role of HPV and intrapulmonary shunting are also discussed earlier $[4,10,14,22]$.

A significant correlation between $\mathrm{ORi}$ and $\mathrm{SpO}_{2}$ was found at time points of DS5, DL5 and, at OLV10. The relationship between $\mathrm{SpO}_{2}$ values and ORi equals to zero values for predicting hypoxemia during anesthesia induction and maintenance is supported by these statistical findings. There are previous studies that support these correlations $[7-9,13,15,17-21]$. In our study group, hypoxemia episodes were observed at various time points throughout the surgery however, the reports were not able to demonstrate a fall of pulse oximeter values below $95 \%$ as $\mathrm{FiO}_{2}$ values were set at $50 \%$ and may have been rised up to $70 \%$ after anesthesia management throughout the surgical procedures. In addition to temporary rises in $\mathrm{FiO}_{2}$ throughout surgery, mechanical ventilation and anesthetic maneuvers were performed by the anesthesiologists. Because of these interventions, in our opinion, we were not able to show a continuous a correlation between ORi and $\mathrm{SpO}_{2}$ values at all measured time points. When ORi which is an oximeter-related parameter is used along with the pulse oximeter monitoring, ORi values may present and record early signs of the downward trend of $\mathrm{PaO}_{2}$ in comparison to a pulse oximetry value. In a previous study, at $1 \mathrm{~min}$ after start of OLV the measurements show that; hypoxemia was $27.5 \%$ where $\mathrm{SpO}_{2}$ value was less than $90 \%$ whereas; a negative predictive value was reported as $12.9 \%$ in those patients who did not achieve an ORi value of 0 at $1 \mathrm{~min}$ after the lung collapsed. It has been reported that median time until desaturation was approximately 5.5 to $6 \mathrm{~min}$. Therefore, $\mathrm{FiO}_{2}$ values should be kept between 50 to $60 \%$ to avoid hyperoxemia and its related adverse effects such as atelectasis $[7-9,13,17,18,20,21]$.
Our findings show similarity with a recent study by Alday and his colleagues [8] however, they also suggested that these values may be used to prevent unnecessary hyperoxemia. In our study, it is clear that during anesthetic management $\mathrm{FiO}_{2}$ values are kept at a value of 50 to $70 \%$ in our patients whereas other studies investigated the use of ORi for hyperoxemia as well $[7-9,13,17$, $18,20,21]$. In a study by Applegate and his colleagues, a positive correlation between ORi values and $\mathrm{PaO}_{2}$ values of $240 \mathrm{mmHg}$ or lower $(r=0.536, p<0.01)$ in comparison to ORi values and $\mathrm{PaO}_{2}$ values of higher than $240 \mathrm{mmHg}$ $(r=0.0016, p>0.05)$ [9]. In our study, we were not able to measure $\mathrm{PaO}_{2}$ values on each time point because of hospital policies to decrease medical costs. In our study, at the measurement time of arterial blood gas analysis at DL5, we found that 4 patients had a $\mathrm{PaO}_{2}$ value above $240 \mathrm{mmHg}$ and ORi values showed statistically significant negative correlation $(r=-1.0, p<0.001)$. In another study, 15 patients undergoing elective thoracic surgery using OLV were evaluated for correlation between $\mathrm{PaO}_{2}$ and ORi parameters throughout the surgical procedure and showed that ORi has a significant correlation with $\mathrm{PaO}_{2}(r=0.671, p<0.001)$ [18]. There are a few studies that provide evidence that $\mathrm{PaO}_{2}$ values show positive correlation with ORi values $[7,9,11,18,20,21]$.

During pulse oximetry monitoring, there is a sigmoidal relationship between arterial oxygenation in blood gas value and peripheral oxygenation reported as $\mathrm{SpO}_{2}$ value on the pulse oximetry device. This relationship causes no change in pulse oximeter values until $\mathrm{PaO}_{2}$ falls below $80 \mathrm{mmHg}$. Afterward, there is a sudden drop in pulse oximetry value; however, the $\mathrm{PaO}_{2}$ is unacceptable for more than 3 to $5 \mathrm{~min}$. Therefore, there is a need to investigate a larger scale of several wavelengths to detect quantitative measurement of methemoglobin, carboxyhemoglobin, and total hemoglobin, and a newly presented device achieved this. Masimo Rainbow Signal Extraction Technology introduced the device [14-16, 19]. ORi is a parameter-driven from this device that is between 0 and 1 values, and it is sensitive to the changes in arterial oxygenation in the blood, with the range of 100 to $200 \mathrm{mmHg}[2,7-9,13,15,18,20,21]$. When oxygenation is in the moderate hyperoxic content showing an arterial blood oxygenation value of $100-240 \mathrm{mmHg}$ in arterial blood gas analysis, the pulse oximeter $\mathrm{SpO}_{2}$ value remains $100 \%$, whereas, there is a decrease in the value of ORi $[2,7-9,13,18,20,21]$. In our study, Fig. 1 and Table 4 provides data on time-dependent correlations between ORi with $\mathrm{SpO}_{2}$.

Increased intrathoracic pressure with respiration leads to more immediate reductions in peripheral perfusion in patients with a fluid deficit. In this case, a decrease in the PI value of the patient is observed. As a result of 
these changes with respiration, the highest and lowest PI ratio corresponds to the PVI. High PVI values are observed in patients with a high fluid deficit or those who do not respond to fluid application changes with changes in the PI [11, 12, 15-17, 23, 24]. In our study, we investigated the ORi and PVI values at different time points during anesthesia induction and maintenance of thoracic surgery and our findings are in correspondence with the previous findings that; fluid deficit or fluid overload causes changes in PI and PVI values. This can be observed in our representative trend graphs in Figs. 2 and $3[16-18,23,24]$.

Our study provides valuable data for the investigation of correlations between ORi and PI, and PVI. OLV with DLT has significant cardiopulmonary physiological changes, as has been discussed elsewhere $[14,16,17,19]$. Our study provides data that at a time point of DS5, there is a significant negative correlation with PI $(r=-0.332$, $p=0.019$ ), whereas; no correlations with PVI were noted. This finding is thought to result from anesthesia drugs that are use during anesthesia induction and especially the use of opioid medications [3-6, 10, 12].

The use of $\mathrm{FiO}_{2}$ values higher than $50 \%$ during anesthesia is related to hyperoxemia, and this high oxygenation decreases cardiac output by reducing heart rate and causing systemic vasoconstriction. Furthermore, hyperoxemia is a potent vasoconstrictor stimulus to the coronary circulation, functioning at the level of the microvascular resistance vessels $[7,21]$. Tsuchiya et al. demonstrated that the PVI could be used to evaluate hypotension that is caused secondary to anestethic drugs in patients undergoing general anesthesia without age group classification [23]. This technique has been used in patients undergoing mechanical ventilation in the intensive care unit to detect fluid responsiveness through respiratory patterns and peripheral perfusion changes [11]. There are insufficient data to distinguish the cause of hypotension due to peripheral vasodilatation and fluid redistribution or cardiac output decrease after general anesthesia [23, 24]. High PVI values are observed in patients with a high fluid deficit or those who do not respond to fluid application changes with changes in the PI [24].

In our study, we demonstrated a time-dependent correlation between PVI and MAP at the time point of OLV90, indicating that PVI showed a relation to MAP at a late stage of the surgical procedure. Recently, it is pointed out in a meta-analysis that PVI is a reliable marker in evaluating a response to fluid management [16].

\section{Limitations}

Malpositioning of DLT may cause hypoxemia and, in our study protocol, we included these patients and therefore, this is a limitation of our study $[8,9,13,25]$. Previously, the arterial blood gas oxygenation results show that $\mathrm{PaO}_{2}$ values were higher during right-sided OLV than left-sided OLV. Although it could be predicted that ORi would decrease before the decrease in $\mathrm{SpO}_{2}$ during left-sided OLV, the actual extent of this application of either right-sided or left-sided OLV needs to be further evaluated $[7,16,18,20,21]$. When oxygenation is in the moderate hyperoxic range of $\mathrm{PaO}_{2}$ values between 100 and $240 \mathrm{mmHg}$, ORi decreases, but $\mathrm{SpO}_{2}$ does not [21]. Therefore, ORi values can be used for detection of hyperoxemia however, as we were not able to measure $\mathrm{PaO}_{2}$ values secondary to hospital protocol to decrease medical costs, we were not able to evaluate these findings.

\section{Conclusions}

During use of OLV for thoracic surgeries, from $5 \mathrm{~min}$ after intubation (DS5) up to 30 min after start of OLV, ORi provides valuable information in predicting hypoxemia defined as $\mathrm{SpO}_{2}$ less than $95 \%$ on pulse oximeter at $\mathrm{FiO}_{2}$ higher than $50 \%$. These findings correlated to the previous reports that HPV increases and intrapulmonary shunting decreases after the start of OLV within 30 to $60 \mathrm{~min}$. ORi provides information for impending hypoxemia that a change in ORi value can be detected 5 to 6 min earlier than pulse oximetry value. Therefore, ORi can provide a valuable time to the anesthesiologist to provide an increase in $\mathrm{FiO}_{2}$ values, to perform necessary mechanical ventilation adjustments, to perform aspiration or other anesthetic management techniques to prevent hypoxemia. Fluid responses and anesthesia induction medications has influence over changes in PI and PVI oximeter values. The use of ORi for hyperoxemia during OLV and thoracic surgeries may be useful however, it is not practical as the $\mathrm{PaO}_{2}$ values of these patients usually range between $60 \mathrm{mmHg}$ and $200 \mathrm{mmHg}$ and patients are not under risk of hyperoxemia related problems when compared to a higher possible risk of hypoxemia.

\section{Abbreviations \\ ASA-PS: American Society of Anesthesiologists Physical Status; Cl: Confidental interval; DBP: Diastolic blood pressure; DLT: Double lumen tube; $\mathrm{FiO}_{2}$ : Fraction of inspired oxygen; IQR: Interquartile range; ORi: Oxygen reserve index; OLV: One-lung ventilation; $\mathrm{PaCO}_{2}$ : Arterial partial pressure of carbon dioxide; $\mathrm{PaO}_{2}$ : Arterial partial pressure of oxygen; PI: Perfusion index; PVI: Pleth variability index; $\mathrm{SpO}_{2}$ : Peripheral oxygen saturation; VATS: Video-assisted thoracos- copy; MAP: Mean arterial pressure; SBP: Systolic blood pressure; SD: Standard deviation.}

\section{Acknowledgments}

Authors would like to thank all the patients for their willingness to participate in the study and their patience.

\section{Authors' contributions}

Concept - G.S., Y.A.K.; Design - G.S., Y.A.K.; Supervision - G.S., Y.A.K.; Data Collection and/or Processing - G.S., Y.A.K.; Analysis and/or Interpretation - G.S.; 
A.B.; Literature Review - G.S., Y.A.K., A.B.; Writer - G.S., Y.A.K; A.B.; Critical Review - G.S., A.B. The author(s) read and approved the final manuscript.

\section{Funding}

The authors received no financial support for the research and/or authorship of this article. This study was done solely by the funding of in Anesthesiology and Reanimation Department of Trakya University Trakya School of Medicine Hospital, Edirne, Turkey.

\section{Availability of data and materials}

The data is available by permission from Dr. Gonul Sagiroglu, Trakya University, School of Medicine, Depertment of Anesthesiology, Edirne, Istanbul, email address: gonul.sagiroglu45@gmail.com. Please contact this address for permission. Administrative permissions are not required to access the raw data. The authors have agreed to give permission to the data and materials during registration at clinicaltrials.gov.

\section{Declarations}

\section{Ethics approval and consent to participate}

All procedures performed in studies involving human participants were in accordance with the ethical standards of the institution and/or national research committee. Informed consent was obtained from all individual participants included in the study.

Written informed consent was obtained from all patients.

\section{Consent for publication}

Not Applicable.

\section{Competing interests}

The authors declared no conflicts of interest with respect to the authorship and/or publication of this article.

\section{Author details}

'Department of Anesthesiology and Reanimation, Trakya University Faculty of Medicine, Edirne, Turkey. ${ }^{2}$ Pendik District Hospital, Clinic of Anesthesiology and Reanimation, Pendik, 34980 Istanbul, Turkey. ${ }^{3}$ Department of Thoracic Surgery, Trakya University Faculty of Medicine, Edirne, Turkey.

Received: 18 October 2021 Accepted: 7 December 2021 Published online: 20 December 2021

\section{References}

1. Lohser J, Slinger P. Lung injury after one-lung ventilation: a review of the pathophysiologic mechanisms affecting the ventilated and the collapsed lung. Anesth Analg. 2015;121:302-18. https://doi.org/10.1213/ANE.00000 00000000808 .

2. Campos JH, Sharma A. Predictors of hypoxemia during one-lung ventilation in thoracic surgery: is oxygen reserve index (ORi) the answer? J Cardiothorac Vasc Anesth. 2020;4:423-5. https://doi.org/10.1053/j.jvca.2019.08.017.

3. Karzai W, Schwarzkopf K. Hypoxemia during one-lung ventilation: prediction, prevention, and treatment. Anesthesiology. 2009;1 10:1402-11. https:// doi.org/10.1097/ALN.0b013e31819fb15d.

4. Yoon S, Kim BR, Min SH, Lee J, Bahk JH, Seo JH. Repeated intermittent hypoxic stimuli to operative lung reduce hypoxemia during subsequent one-lung ventilation for thoracoscopic surgery: a randomized controlled trial. PLoS One. 2021;16(4):e0249880. https://doi.org/10.1371/journal.pone. 0249880 .

5. Hahm TS, Jeong H, Ahn HJ. Systemic oxygen delivery during one-lung ventilation: comparison between propofol and sevoflurane anaesthesia in a randomised controlled trial. J Clin Med. 2019;8:1438. https://doi.org/10. 3390/jcm8091438.

6. Liu TJ, Shih MS, Lee WL, et al. Hypoxemia during one-lung ventilation for robot-assisted coronary artery bypass graft surgery. Ann Thorac Surg. 2013;96:127-32. https://doi.org/10.1016/j.athoracsur.2013.04.017.

7. Chen ST, Min S. Oxygen reserve index, a new method of monitoring oxygenation status: what do we need to know? Chin Med J (Engl). 2020;133:229-34. https://doi.org/10.1097/CM9.0000000000000625.
8. Alday E, Nieves JM, Planas A. Oxygen reserve index predicts hypoxemia during one lung ventilation. An observational diagnostic study. J Cardiothorac Vasc Anesth. 2020;34:417-4. https://doi.org/10.1053/j.jvca.2019.06.035.

9. Applegate RL 2nd, Dorotta IL, Wells B, Juma D, Applegate PM. The relationship between oxygen reserve index and arterial partial pressure of oxygen during surgery. Anesth Analg. 2016;123:626-33. https://doi.org/10.1213/ ANE.0000000000001262.

10. Marongiu I, Spinelli E, Mauri T. Cardio-respiratory physiology during onelung ventilation: complex interactions in need of advanced monitoring. Ann Transl Med. 2020;8:524. https://doi.org/10.21037/atm.2020.03.179.

11. Scheeren TWL, Belda FJ, Perel A. The oxygen reserve index (ORi): a new tool to monitor oxygen therapy. J Clin Monit Comput. 2018;32:379-89. https:// doi.org/10.1007/s10877-017-0049-4.

12. Loupec T, Nanadoumgar $H$, Frasca D, et al. Pleth variability index predicts fluid responsiveness in critically ill patients. Crit Care Med. 2011;39:294-9. https://doi.org/10.1097/CCM.0b013e3181ffde1c.

13. Durkin C, Romano K, Egan S, Lohser J. Hypoxemia during one-lung ventilation: does it really matter? [published online ahead of print, $2021 \mathrm{Jul}$ 7]. Curr Anesthesiol Rep. 2021:1-7. https://doi.org/10.1007/s40140-021-00470-5.

14. Nagendran J, Stewart K, Hoskinson M, Archer SL. An anesthesiologist's guide to hypoxic pulmonary vasoconstriction: implications for managing singlelung anesthesia and atelectasis. Curr Opin Anaesthesiol. 2006;19:3. https:// doi.org/10.1097/01.aco.0000192777.09527.9e.

15. Yuksek A. Utility of the pleth variability index in predicting anesthesiainduced hypotension in geriatric patients. Turk J Med Sci. 2021;51:134-9. https://doi.org/10.3906/sag-1912-132.

16. Jia FJ, Yan QY, Sun Q, Tuxun T, Liu H, Shao L. Liberal versus restrictive fluid management in abdominal surgery: a meta-analysis. Surg Today. 2017;47:344-56. https://doi.org/10.1007/s00595-016-1393-6.

17. Tusman G, Bohm SH, Suarez-Sipmann F. Alveolar recruitment maneuvers for one-lung ventilation during thoracic anesthesia. Curr Anesthesiol Rep. 2014;4:160-9. https://doi.org/10.1007/s40140-014-0054-9.

18. Koishi W, Kumagai M, Ogawa S, Hongo S, Suzuki K. Monitoring the oxygen reserve index can contribute to the early detection of deterioration in blood oxygenation during one-lung ventilation. Minerva Anestesiol. 2018;84:1063-9. https://doi.org/10.23736/S0375-9393.18.12622-8.

19. Ishida Y, Okada T, Kobayashi T, Uchino H. ORi ${ }^{\mathrm{TM}}$ : a new indicator of oxygenation. J Anesth. 2021;35(5):734-40. https://doi.org/10.1007/ s00540-021-02938-4.

20. Yoshida K, Isosu T, Noji Y, et al. Usefulness of oxygen reserve index $\left(O R i^{\mathrm{TM}}\right)$, a new parameter of oxygenation reserve potential, for rapid sequence induction of general anesthesia. J Clin Monit Comput. 2018;32:687-91. https:// doi.org/10.1007/s10877-017-0068-1.

21. Yoshida K, Isosu T, Noji Y, et al. Adjustment of oxygen reserve index $\left(\mathrm{OR}^{\mathrm{TM}}\right)$ to avoid excessive hyperoxia during general anesthesia. J Clin Monit Comput. 2020;34(3):509-14. https://doi.org/10.1007/s10877-019-00341-9.

22. Habre W, Peták F. Perioperative use of oxygen: variabilities across age. Br J Anaesth. 2014;113(Suppl 2):ii26-36. https://doi.org/10.1093/bja/aeu380.

23. Tsuchiya M, Yamada T, Asada A. Pleth variability index predicts hypotension during anesthesia induction. Acta Anaesthesiol Scand. 2010;54:596-602. https://doi.org/10.1111/j.1399-6576.2010.02225.x.

24. Canneson M, Desebbe $O$, Rosamel P, et al. Pleth variability index to monitor the respiratory variations in the pulse oximeter plethysmographic waveform amplitude and predict fluid responsiveness in the operating theatre. $\mathrm{Br}$ J Anaesth. 2008;101(2):200-6. https://doi.org/10.1093/bja/aen133.

25. Inoue S, Nishimine N, Kitaguchi K, Furuya H, Taniguchi S. Double lumen tube location predicts tube malposition and hypoxaemia during one lung ventilation. Br J Anaesth. 2004;92:195-201. https://doi.org/10.1093/bja/aeh055.

\section{Publisher's Note}

Springer Nature remains neutral with regard to jurisdictional claims in published maps and institutional affiliations. 